\title{
Conditional entropies, phase synchronization and changes in the directionality of information flow in neural systems
}

\author{
Michał Żochowski and Rhonda Dzakpasu \\ Department of Physics and Biophysics, Research Division, University of Michigan, Ann Arbor, \\ MI 48109, USA
}

Received 4 November 2003, in final form 6 February 2004

Published 10 March 2004

Online at stacks.iop.org/JPhysA/37/3823 (DOI: 10.1088/0305-4470/37/12/007)

\begin{abstract}
We devised a novel measure that dynamically evaluates temporal interdependences between two coupled units based on the properties of the distributions of their relative interevent intervals. We investigate its properties on the system of two coupled non-identical Rössler oscillators and a system of non-identical Hindmarsh-Rose models of thalamocortical neurons and show that the measure highlights the properties of phase synchronization observed in those two systems. We postulate that the observed properties of the phase lag, in conjunction with the experimentally observed activity-dependent synaptic modification in the neural systems, may drive the changes of the direction of information flow in a neural network, and thus the measure can play an important role in assessing those changes.
\end{abstract}

PACS numbers: 87.18.Hf, 05.45.Xt, 87.17.Aa, 05.65.Tp, 87.19.La

\section{Introduction}

Synchronization and formation of spatio-temporal patterning have been studied extensively in different systems ([1-5] and references therein). It has been hypothesized that such correlations can play an important role especially in biological systems [6-9]. For example, it has been postulated that synchronization in activity of the neural systems can play a crucial role during information processing [10]. Moreover, it was recently established that relative timings between the spiking of coupled neurons lead differentially to long- and short-term changes in connectivity in the network [11-13]. This is due to the experimentally established fact that synapses connecting presynaptic and postsynaptic neuron will be potentiated if the presynaptic neuron fires a spike within a short window (up to few milliseconds) before the postsynaptic one, and conversely it can be inhibited if the postsynaptic neuron emits a spike just before the presynaptic one. 
To be able to monitor successfully such spatio-temporal patterning in dynamical systems in general it is crucial to develop adequate methods to measure relative timings of the observed events. Any method designed for this purpose must be statistical, based on information obtained from multiple events, but at the same time, able to detect dynamical changes in the system. Most of the methods used to detect temporal correlations between interacting elements are based on cross correlations and provide statistical values of the degree of overlap between the event timings. Those measures are symmetrical in their nature providing an overall level of overlap between the firing patterns, and the small temporal shifts in the correlation peak may be easily left undetected. However, in light of the aforementioned findings (i.e. the change in connectivity depends asymmetrically on the timing of coupled neurons) the direction of those shifts may be of crucial importance to the functioning of the network.

We have developed a novel measure that accounts for this asymmetry in temporal shifts which, as will be shown below, can be used to gain a valuable insight into the activity of the coupled non-identical units. The developed measure is based on the dynamical monitoring of entropy changes in relative firing patterns between two units. We will refer to it as a conditional entropy (CE) since it is calculated based on the relative timings of the events of both coupled units. To show the applicability of the proposed method, we applied the measure to monitor dynamics of a system of two non-identical, coupled units (Rössler oscillators and then models of thalamocortical neurons). Our measure highlighted an important, earlier observed phenomenon-the two non-identical units establish a phase synchronized state [5, 14-19] with a phase lag that varies dramatically with the relative properties of the two units. We link this observation to information processing in the neural systems in the conclusion of the paper.

\section{Conditional entropies-a measure of the properties of the phase synchronization in the coupled system}

After the seminal work of Shannon [20], the entropy measurement is often used in information theory to obtain the statistical properties of a system and is directly linked to its dynamical properties [21-24]. Here, the conditional entropies are calculated for every unit in the coupled pair separately and they measure relative properties of the units with respect to each other. When the information is combined, the entropies effectively monitor the changes in the distributions of phase relationships between the two coupled units.

Here, the phase of the two units is converted into their event timings. The occurrence of the event corresponds to a specific phase value of the unit. Such formulation is particularly useful when the event defining a phase can be easily measured experimentally, i.e. spike of neuron. Thus, to calculate the CEs we create two separate histograms that simultaneously monitor relative interevent durations of the events

- of unit 2 with respect to the events of unit 1 and

- of unit 1 with respect to the events of unit 2 .

The interevent durations $\Delta t_{l m}^{12}$ of unit 2 with respect to unit 1 are calculated as a difference between the timings of the event of unit $2\left(t_{l}^{2}\right)$ and the last event of unit $1\left(t_{m}^{1}\right)$ (i.e. $\left.\Delta t_{l m}^{12}=t_{l}^{2}-t_{m}^{1}\right)$, and conversely the interevent timings $\Delta t_{j k}^{21}$ of unit 1 are calculated as a time difference $\left(\Delta t_{j k}^{12}=t_{j}^{2}-t_{k}^{1}\right)$ with respect to the timing of the last event of unit 2 (see figure 1). Thus, in essence two simultaneous questions are being asked: what are the properties of the distribution of the event timings of unit 2 that occur directly after (and relative to) the latest event of unit 1 , and analogously what are the properties of the distribution of the event timings of unit 1 relative to the latest event of unit 2? Such a definition of the measure highlights 


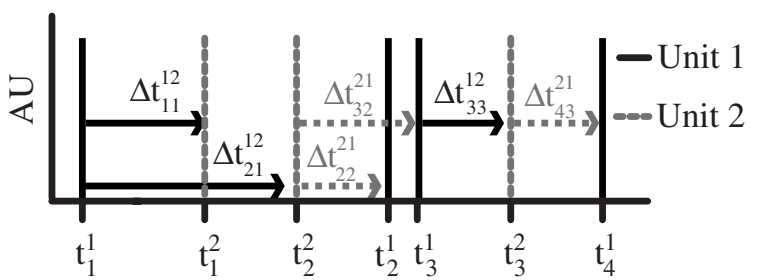

Figure 1. Relative interevent durations used to calculate the conditional entropy. The timings of one unit are calculated with respect to the timing of the last event of the other. This assures asymmetry of the measure with respect to the sign of the relative temporal shift of the events of the two units. The labels $t_{l}^{i}$ on the $x$-axis denote the timing of the $l$ th event appearing on the $i$ th unit $(i \in 1,2)$; the $\Delta t_{l m}^{i j}$ denotes the interevent duration between the timing of the last event on $i$ th unit $\left(t_{m}^{i}\right)$ and the timing of the $l$ th event of the $j$ th unit following it $\left(t_{l}^{j}\right)$.

the asymmetry in the treatment of the relative timing delays, but when combined provides full information about the interaction between the two coupled units. The measure will identify any phase locking appearing between the two units, as the width of the distributions of the interevent times between the units will vary significantly. The unit that is being driven will generate an event with a relatively small and constant delay after the event of the driving unit creating a narrow interevent distribution. On the other hand, the reversed interevent times will depend on the dynamical properties of the driver itself (i.e. patterns of external input) and may vary significantly generating wider distribution in the interevent times.

Additionally, to allow the measure to monitor changes in the distributions of the relative event timings the distributions are updated continuously ('on line'), as the events occur at unit 1 and/or unit 2. Specifically, the distribution of the relative event timings of unit 2 with respect to the last event of unit 1 is updated as the event occurs at unit 2 , and conversely, the distribution of the timings of unit 1 with respect to the timing of the last event of unit 2 is updated at the time the event occurs at unit 1 . The distributions are updated by increasing the bin of the appropriate distribution within which the latest timing falls by a fixed $\Delta P$. Specifically, if $n$th event occurs at time $t_{n}$ so that the relative event timing $\Delta t_{n-1}^{i j}$ is of the length $\Delta T(I-1)<\Delta t_{n-1}^{i j} \leqslant I \Delta T$ and the timing falls within the bin $I$, the cumulative probability of bin $I$ at $t_{n}$ is given by $P_{I}\left(t_{n}\right)=P_{I}\left(t_{n-1}\right)+\Delta P$. The updated distribution is then renormalized. The parameter $\Delta P$ effectively determines the speed with which the distribution is to be updated based on information obtained from the newest events. Therefore, if the properties of the distribution change so that the given bin $Q$ is not updated during last $n$ events, then the probability of that bin at the time of the event $m$ declines to

$$
P_{Q}\left(t_{m}\right)=\frac{1}{(1+\Delta P)^{n}} P_{Q}\left(t_{m-n}\right)
$$

The conditional entropies are calculated from the normalized distributions, $S=$ $-\sum_{I} P_{I} \ln P_{I}$, each time the distributions are updated.

The strength of the measure is that it provides dynamical assessment of the properties of the phase shift based on the event timings. The measure essentially provides separate information about the width of the distributions of relative interevent times between the units, highlighting the possible asymmetry between the two distributions. This information is especially useful in neuroscience where monitoring temporal interdependences between the coupled neurons can yield critical information about pattern formation during information processing. 


\section{Measurement of phase synchronization of two non-identical oscillators}

Synchronization between two identical coupled units has been studied extensively during the last decade (see, for example, [2, 25-27]). However, in many cases even though the dynamics of the systems are driven by the same underlying processes (i.e. they are described by the same sets of equations), the macroscopic properties of their trajectories will be dramatically different and will depend on the sets of control parameters. This is well exemplified in neural systems where the emission of action potentials by neurons is driven by the same underlying processes (appropriate ionic currents), but it is impossible to assume that all the properties of those ionic currents (conductances for example) as well as currents from external inputs will be the same at every neuron. Moreover, it has been established experimentally that the same neuron can change its firing pattern dramatically depending on the cognitive state of the animal [28]. Thus the spatio-temporal patterns formed by non-identical units (i.e. having different values of their control parameters) can be of significant importance.

If those control parameters are different, the coupling of the trajectories between such systems cannot lead to complete synchronization since the systems will have effectively different dynamical properties. It has been shown, however, that two coupled non-identical chaotic oscillators can achieve phase synchronization - the state when their relative phases are locked but their amplitudes vary $([1,14,15,17,19]$ and references therein). The properties of the obtained phase lag during the phase synchronization vary with the relative frequencies of the coupled units and the coupling strength.

We tested our measure on a system of coupled Rössler oscillators [29] that differ via the value of their control parameters that influence the dynamical properties of their trajectories, and then applied it to a system of two coupled Hindmarsh-Rose models of spiking neurons [30]. Depending on the values of the control parameters the trajectory of both systems can be chaotic or periodic. However, since in both examples the units are oscillatory systems, one can define a temporal phase angle and average frequency [5, 15].

\subsection{Two coupled non-identical Rössler oscillators}

The events for the Rössler oscillator were defined as the times at which the oscillator's trajectory (unit 1 or 2 ) crosses a specified Poincare section $(z=1)$. The equations for the coupled Rössler system are

$$
\begin{aligned}
& \dot{x}_{1,2}=-\left(z_{1,2}+y_{1,2}\right) \\
& \dot{z}_{1,2}=b+\left(x_{1,2}-c\right) z_{1,2}
\end{aligned} \quad \dot{y}_{1,2}=x_{1,2}+a_{1,2} y_{1,2}+\alpha\left(y_{2,1}-y_{1,2}\right)
$$

where the subscript denotes the oscillator number; $a_{1,2}, b=0.2, c=10.0$ are the control parameters of the oscillators and $\alpha=0.4$ is the coupling. The values of parameters $a_{1}$ and $a_{2}$ were different for both units.

We found that our measure highlighted an important result. Within the same numerical integration of the coupled units, the CE of the oscillator with a larger control parameter $\left(a_{i}\right)$ is close to zero, whereas the $\mathrm{CE}$ of the oscillator with lower value of $a_{i}$ is significantly higher (figure 2(A)). Moreover, a small change in the values of the control parameters from $a_{1}>a_{2}$ to $a_{1}<a_{2}$ generates a dramatic change in the values of conditional entropies (figures $2(\mathrm{~A})$ and (B)). Both conditional entropies are equal to zero when $a_{1}=a_{2}$ (the two units are identical). Such behaviour of the CEs is due to the fact that if the coupling is strong enough the two coupled non-identical units (the case when $a_{1} \neq a_{2}$ ) achieve phase synchronization [14, 15]. Here, the value of the phase is represented by the timing of the defined event. Thus the quantity that is being measured is not the value of the phase at the given moment in time, but the timing when the specific value of the phase was achieved. 

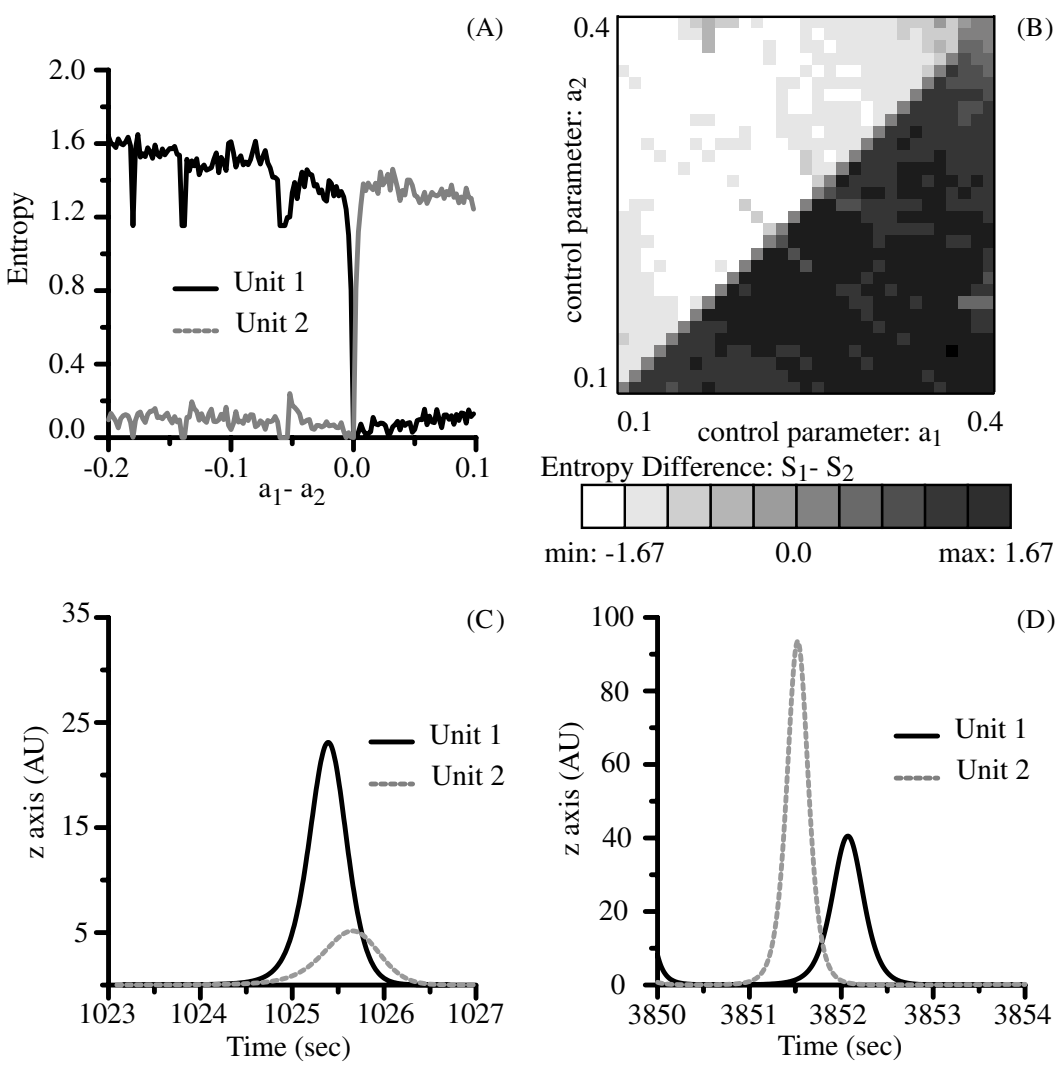

Figure 2. Conditional entropy calculated for two Rössler oscillators with different values of control parameters $\left(a_{1,2}\right)$. Panel A: $a_{1}$ is varied from 0.1 to 0.4 and the control parameter of unit 2 is fixed at $a_{2}=0.3$; the coupling constant $\alpha=0.4$. Panel B: a greyscale map of the conditional entropy difference when both $a_{1,2}$ are varied from 0.1 to 0.4 . Panels $\mathrm{C}$ and $\mathrm{D}$ : the relative timings of the events (crossings of the Poincare section $z=1$ ) for the two units when (C) $a_{1}>a_{2}$ and (D) $a_{2}>a_{1}$.

As has been established earlier [14, 15], the phase at which the two units synchronize depends on the relative values of their average frequencies that in turn are determined by the relative values of their control parameters. The phase of the unit with the higher value of the control parameter will precede that of the unit with the lower value of $a_{i}$ (see figures $2(\mathrm{C})$ and (D)) i.e. the event of the unit with the higher $a_{i}$ will precede that of the unit with lower one. This shift does not change significantly during the evolution of the two units. Since the distributions used to estimate CEs are based on those relative interevent times, the distribution of those interevent durations of unit having lower value of $a_{i}$ with respect to unit having higher one will have a narrow peak corresponding to the value of the phase shift - the entropy of such distribution will tend to zero. On the other hand, the distribution of the events of the unit with smaller $a_{i}$ will be significantly wider since the next event timing will be loosely correlated with the timing of the previous one-the $\mathrm{CE}$ will retain significantly higher value.

For the case when $a_{1}=a_{2}$ (identical units) the complete synchronization is obtained (as expected) and thus the phase shift for both units is equal to zero.

We measured the existence of this property of the phase shift for extended number of $a_{i}$ pairs of the coupled units. Figure 2(B) shows a greyscale map representing the difference 


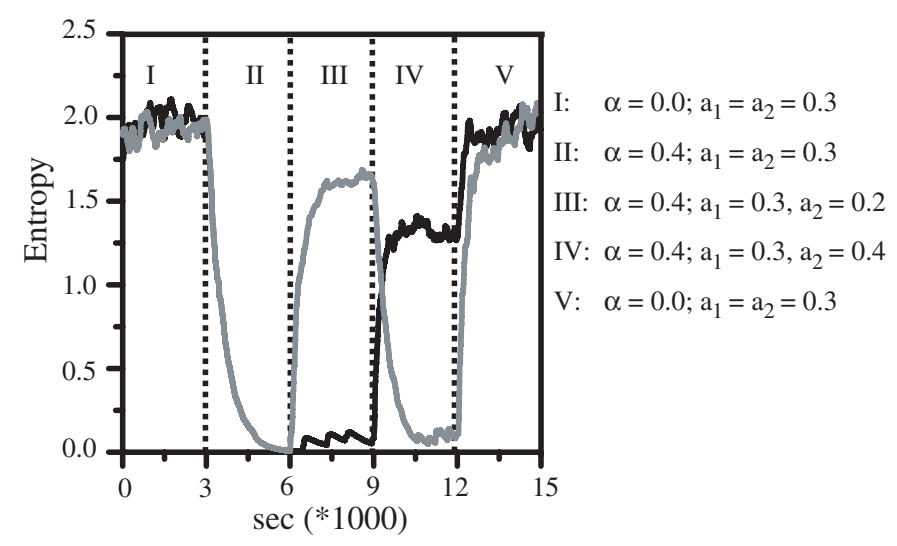

Figure 3. The changes of conditional entropy are monitored during different phases of the same integration. The integration lasted for $15000 \mathrm{~s}$ and included five sections of $3000 \mathrm{~s}$ each. The settings for every section are listed in the figure. The values of the CEs change rapidly as the relations between control parameters of the coupled units change.

between the conditional entropies $\left(S_{1}-S_{2}\right)$; white denotes the minimal value whereas black denotes the maximal one. The $x$-axis denotes the value of the control parameter of unit one $\left(a_{1}\right)$; the $y$-axis corresponds to the value of the control parameter of the other unit $\left(a_{2}\right)$. Since the CEs will detect the direction of the shift and not its value, the values of the difference do not change significantly except along the diagonal $\left(a_{1}=a_{2}\right)$. On the diagonal their difference effectively switches sign. This indicates that the direction of the phase shift depends solely on the relative values of the control parameters of the coupled units.

To illustrate the changes of the $\mathrm{CE}$ when the phase relations between the units dynamically change, we integrated the trajectories of the two oscillators for $15000 \mathrm{~s}$ (figure 3). The relative values of the control parameters, $a_{i}$, are changed every $3000 \mathrm{~s}$. As the relative values of $a_{i}$ change, the conditional entropies change dramatically following the changes in the phase lag of the two units:

- $\alpha=0$ in I and V sections - the two units are independent and both have high conditional entropies;

- $\alpha=0.4$ and $a_{1}=a_{2}$ in section II-the conditional entropies of both units decrease to zero (complete synchronization is obtained);

- $\alpha=0.4$ and $a_{1} \neq a_{2}$ (sections III and IV) - the conditional entropies of the two units change rapidly at the onset of the sections, with the CE of the unit with higher value of $a_{i}$ approaching zero, and that of the other unit retaining significantly higher value (phase synchronization with the phase lag depending on the relative values of control parameters is obtained).

The rate of change of the CEs at the start of every section is determined by the rate of distribution updates, $\Delta P$.

We investigated the behaviour of the proposed measure as a function of the coupling $\alpha$ (figure 4). As in the calculations performed for figure 2, the control parameter of one unit was kept constant and that of the second unit was varied. When the coupling is equal to zero both oscillators are independent, there is no phase relation and thus their conditional entropies are high (figure 4(A)). However, the described timing interdependences materialize even for small couplings. For a coupling as low as $\alpha=0.2$ the conditional entropies for both oscillators 

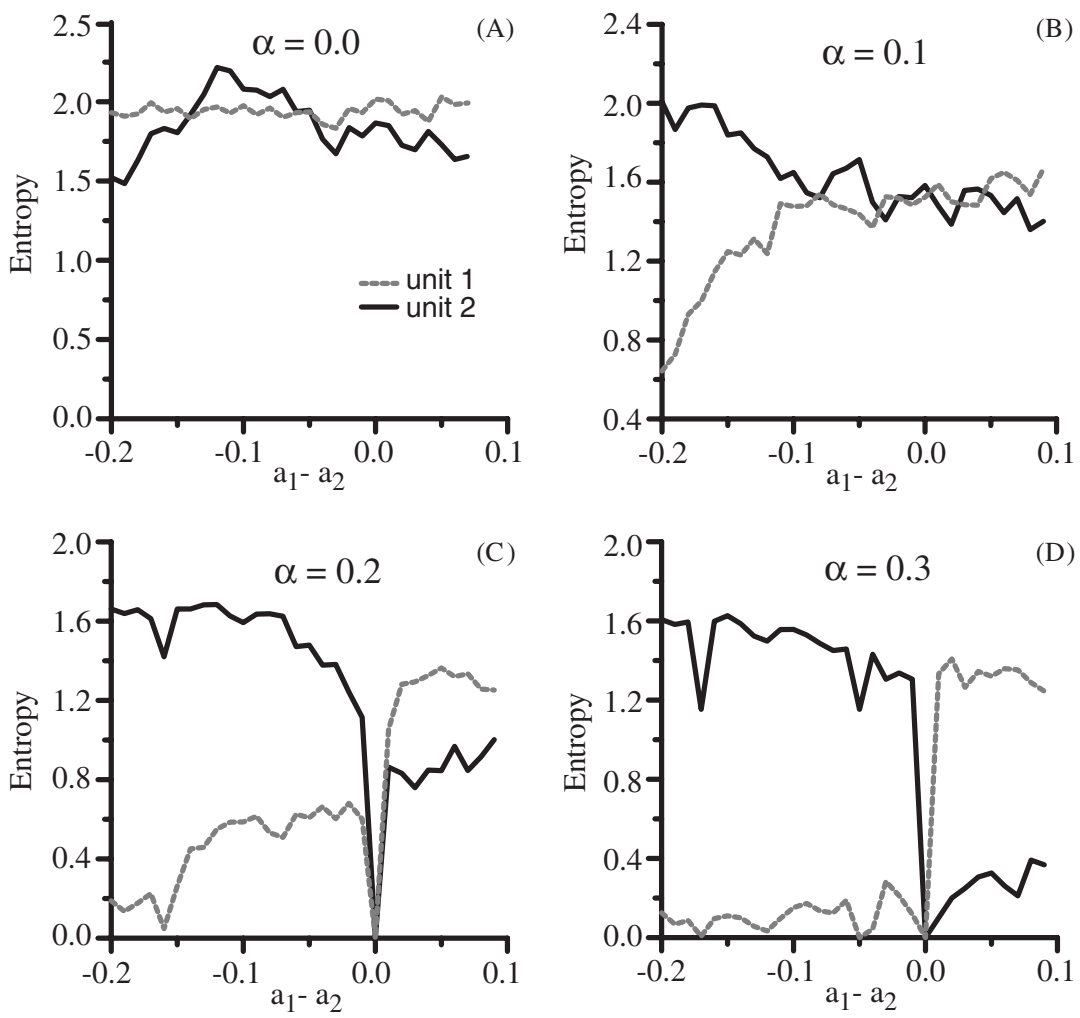

Figure 4. The changes of the conditional entropy in the presence of the coupling of different strengths. The control parameter $a_{1}$ was varied from 0.1 to $0.4, a_{2}=0.3$ and was fixed. The coupling strength $\alpha$ is denoted on top of every panel.

are significantly different (figure 4(B)) even though they are not zero, indicating that the phase synchronization is not complete and the phase fluctuates considerably. The conditional entropies $S_{1}=S_{2}$ and are equal to zero, when both oscillators have the same values of their control parameters (figure $4(\mathrm{C})$ ), indicating complete synchronization. At larger couplings the phase synchronization is obtained and the entropies take their corresponding values (figure 4(D)).

Finally, we investigated robustness of the measure in the presence of noise. The added noise could represent in experimental conditions random jitter associated with dynamics of the experimental system or error of the measurement. A term $A \xi_{1,2}(t)$ was added to the $y$-coordinate of each of the oscillators. The amplitude, $A$, was varied from 0 to 10 and $\xi(t) \in[-1,1]$ was a randomly generated, uniformly distributed variable. The observed phase shift was relatively robust with respect to noise (figure 5). However the transition region around the point where $a_{1}=a_{2}$ widened considerably as the noise increased (figures 5(B), (C) and (D)). This is due to the fact that the magnitude of the phase lag is proportional to the difference of the control parameters of the coupled units. For similar values of $a_{i}$ the shifts are relatively small and the applied noise can randomize event order (i.e. the event of a unit with smaller $a_{i}$ will sometimes take place before that of the unit with larger $a_{i}$ ). For larger phase shifts, phase variation due to noise in not large enough to randomize the order of the measured events. 

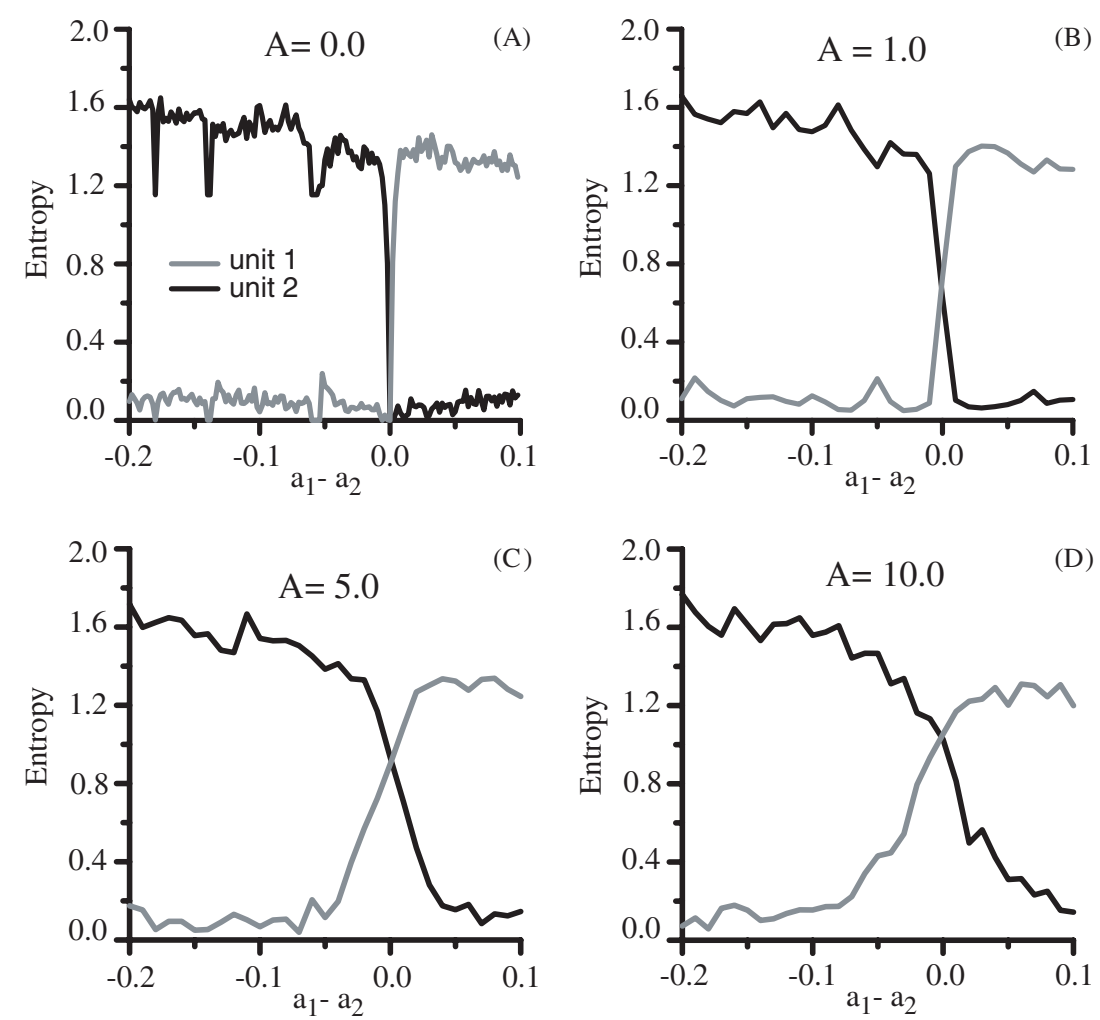

Figure 5. Stability of the relative timings of the events in the presence of noise. The conditional entropy is measured for different relative values of the control parameters of the coupled Rössler oscillators (as before $a_{1}$ was varied from 0.1 to $0.4, a_{2}=0.3$ and was fixed). The amplitude of noise, $A$, is marked on each panel. The region of $\mathrm{CE}$ transition widens considerably.

\subsection{Two coupled models of thalamocortical neurons}

We also applied our measure on the Hindmarsh-Rose model of thalamocortical neurons [30-32]. The equations for the coupled Hindmarsh-Rose neurons are

$$
\begin{array}{ll}
\dot{x}_{1,2}=y_{1,2}-a x_{1,2}^{3}+b x_{1,2}^{2}-z_{1,2}+I_{0_{1,2}}+\alpha\left(x_{2,1}-x_{1,2}\right) & \dot{y}_{1,2}=c-d x_{1,2}^{2}-y_{1,2} \\
\dot{z}_{1,2}=r\left[s\left(x_{1,2}-x_{0}\right)-z_{1,2}\right] &
\end{array}
$$

where the subscript denotes the neuron number; $a=1.0, b=3.0, c=1.0, d=5.0, r=$ 0.006, $s=4.0$ and $x_{0}=-1.6$ are the parameters of the model and $\alpha=1.1$ is the coupling strength. The parameter $I_{0_{i}}$ represents the amplitude of the external current applied to the neuron and is the control parameter of the system. We performed measurements of conditional entropies for the two coupled neurons. Here, the event timings were naturally chosen to be the timings of action potentials emitted by the neurons. As before, the control parameter $I_{0_{i}}$ of the two neurons was varied (figure 6). The conditional entropies changed in the same manner as in the case of the Rössler oscillators, indicating the formation of a phase synchronization between the two neurons (figures 6(C) and (D)). As before the direction of the phase shift depended only on the relative values of the control parameters (i.e. relative frequencies of the coupled neurons) and again appeared over the whole range of the control parameter pairs (figure 6(B)). The grey scale denotes the difference between the conditional entropies of the two neurons; 

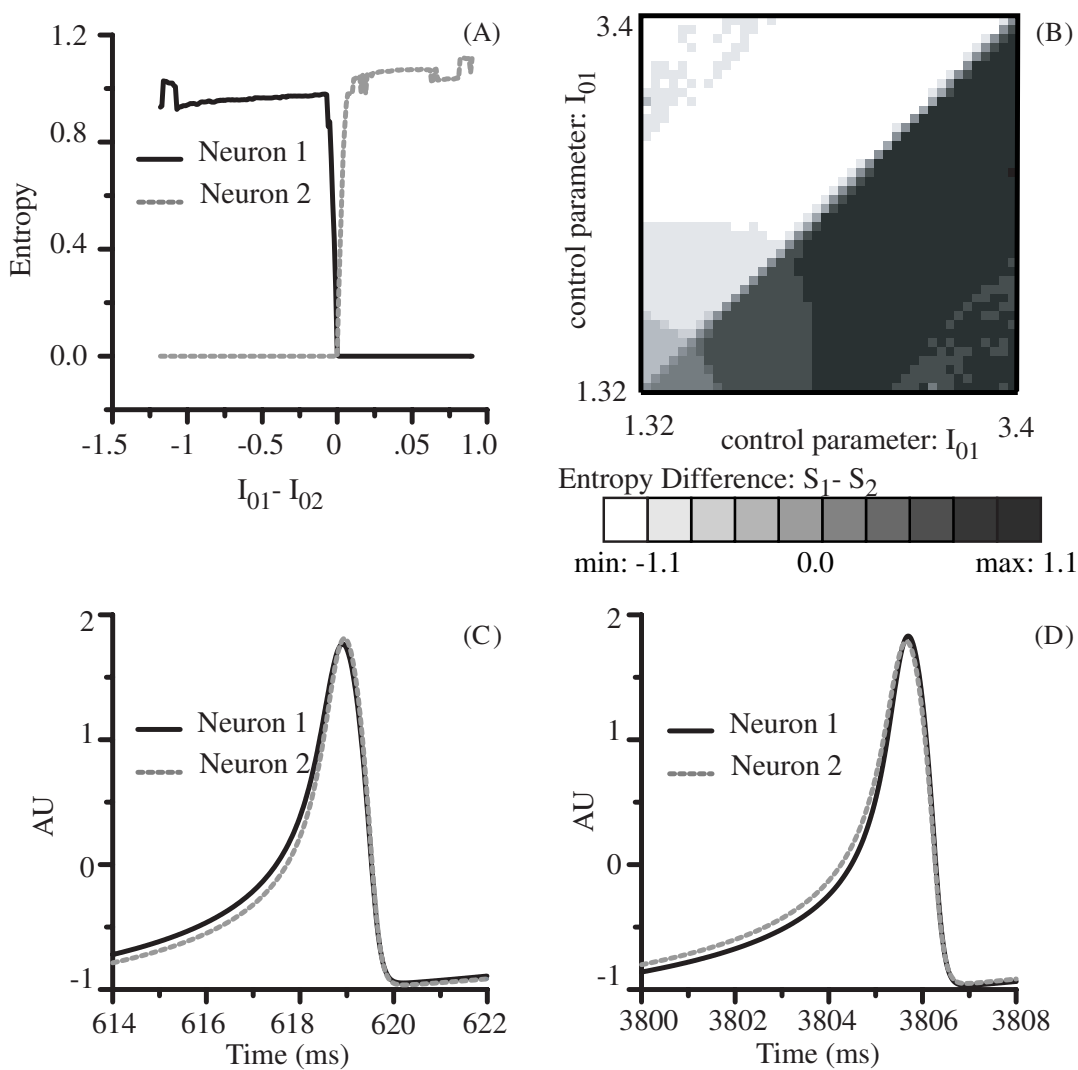

Figure 6. Conditional entropy calculated for two Hindmarsh-Rose neurons with different relative levels of the external current (value of $I_{0_{1,2}}$ ). Panel A: $I_{0_{1}}$ is varied from 1.32 to 3.4 and the control parameter of neuron 2 is fixed at $I_{0_{2}}=2.5$. Panel B: a greyscale map of the conditional entropy difference when both $I_{0_{1,2}}$ are varied from 1.32 to 3.4. Panels $C$ and $D$ : the relative timings of the spikes for the two neurons when (C) $I_{0_{1}}>I_{0_{2}}$ and (D) $I_{0_{2}}>I_{0_{1}}$.

$x$-axis denotes the values of the control parameter of neuron $1\left(I_{0_{1}}\right) ; y$-axis denotes the values of $I_{0_{2}}$.

\section{Conclusions-implications for information processing in neural systems}

In conclusion, we have constructed a measure that dynamically monitors the changing properties of phase synchronization in two coupled units. The method is based on a separate measurement of the distributions of relative interevent durations occurring in the two coupled systems and essentially identifies and compares the temporal structure of the changes of their phase shifts over time. At the same time, this method highlights a possible asymmetry between the two patterns. We have shown properties and the sensitivity of the measurement for two coupled Rössler oscillators and the Hindmarsh-Rose model of spiking neurons. Our measure has highlighted the earlier established fact $[14,15]$, that the properties of the phase synchronization between two coupled oscillatory units depend critically on the relative frequencies between the two. The unit with the lower frequency will have a stable positive phase lag with respect to the one with higher frequency. This phase lag is represented as a difference of event timings that in turn denotes a specific phase of the unit (Poincare section in 
the case of Rössler oscillators and timing of an action potential in the case of the HindmarshRose model of a neuron). The value of the conditional entropy is highly sensitive to the directionality of the phase lag-it converges to zero if the phase lag of one unit is positive, and thus consistent with respect to that of the other unit and approaches relatively higher value otherwise. We have also shown that the measure performs well in the presence of noise indicating its applicability to experimental measurement.

Our proposed method could be particularly useful in detecting interdependences between neural populations. Applications of our measure to identify temporal interdependences of the spike trains of two neurons may lead to increased understanding of spike timing dependent changes in the network structure. We postulate that the properties of the phase shift during a relatively weak event of the phase synchronization can be of significant importance to the functioning of neural systems.

It has already been shown that synchronization is prevalent in the neural systems. Furthermore, it has been established that changes in connectivity in a network (through synaptic modification) depend crucially on the relative timings of the action potentials emitted by the coupled neurons. Specifically, it was experimentally established that synaptic longterm potentiation (LTP) and long-term depression (LTD) as well as the short-term changes in synaptic strength are directly linked to the relative timings of the emitted spikes [11-13]. That is, if the presynaptic neuron emits a spike in a narrow window before the postsynaptic neuron the connecting synapse will be potentiated. Conversely, if the postsynaptic neuron emits a spike systematically before the presynaptic neuron the corresponding synapse will be depressed. Therefore, if two neurons are reciprocally coupled (as it commonly happens in the neural systems) having two synapses (from neuron 1 to neuron 2 and a separate one from neuron 2 to neuron 1 ) and if neuron 1 receives a higher synaptic input than neuron 2 (as in the case of the Hindmarsh-Rose example) the synapse mediating depolarization from neuron 1 to neuron 2 will be potentiated over time and the other one depressed. However, if the relative input strength changes so that now neuron 2 receives higher synaptic input than neuron 1 the situation is reversed-the synapse mediating signals from neuron 2 to neuron 1 will be potentiated whereas the other one will undergo depression.

We have performed a simple simulation to illustrate this point and the results are shown in figure 7. We allowed the coupling strengths, $\alpha_{1,2}$, between two Hindmarsh-Rose neurons (in equation (3)) to become independent variables that are linked to respective conditional entropies. The general form of those changes is given by

$$
\alpha_{1,2}=\frac{\lambda}{1+\beta S_{1,2}^{2}}
$$

where $\lambda=1.1$ and $\beta=5.0$ are constants. Additionally, the coupling strengths are bounded so that $\alpha_{1,2} \in[0.2,1.2]$. Initially, the neurons have the same coupling $\left(\alpha_{1,2}=1.1\right)$, but different external inputs, $I_{01}$ and $I_{02}$ (as indicated in figure 7). The coupling $\alpha_{1}$ from the neuron with higher input became progressively stronger whereas that of the other one became significantly smaller until the bounding values were achieved. At $t=3000 \mathrm{~ms}$ the external input to the neurons was changed, so that the neuron with the previously lower input now received a stronger input. The change evoked rapid synaptic modifications (changes in the coupling strength $\alpha_{1,2}$ ) driven by the changing conditional entropies, and thus by the properties of relative timings between the spike times of the two neurons. When steady state was again achieved the synapses effectively reversed their efficacy. Thus the neuron that was initially driven became a driver (figures 7(A) and (B)) - the information flow is reversed.

It is important to note that the goal of this simulation is to show the possible relevance of the properties of the phase lag during phase synchronization achieved by two neurons 

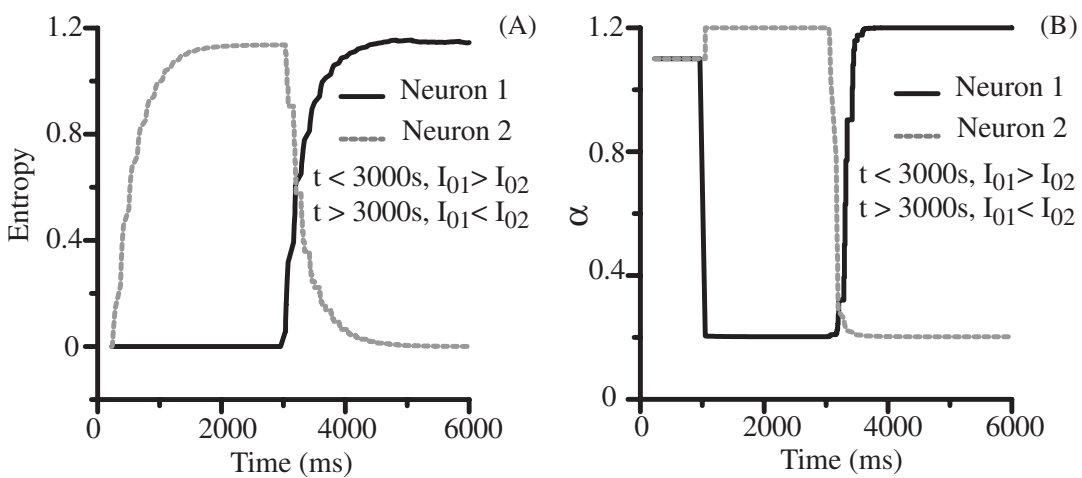

Figure 7. Simulation of the modifications of the non-symmetrical coupling between the neurons with changing input strengths. The information flow reverses itself as the relative input strengths are changed: (A) conditional entropies and (B) coupling strengths $(\alpha)$. The input strength of the two units is switched at $t=3000 \mathrm{~ms}$.

by inducing structural changes in the network and additionally the possible application of conditional entropies to identify them. In neural systems, these synaptic modifications are achieved by complex molecular processes.

These results imply that the network can use properties of phase synchronization to alter its structure during information processing and, if the synaptic modifications due to those timing relations are large enough the direction of the information flow in the network can be altered. Essentially, with a minor change in internal or external environment the master-slave relation between the two neurons can be reversed - the postsynaptic neuron can become a presynaptic one and drive the neuron that was driving it earlier. Thus, in fact the informational content (the external current differentially stimulating appropriate neurons), or internal modifications of the ionic currents can alter the information flow in the network.

The postulated hypothesis will now be investigated experimentally.

\section{References}

[1] Parlitz U, Kocarev L, Stojanovski T and Preckel H 1996 Encoding messages using chaotic synchronization Phys. Rev. E $\mathbf{5 3} 4351$

[2] Rulkov N F, Suschik M M, Tsimring L S and Abarbanel H D I 1995 Generalized synchronization of chaos in directionally coupled chaotic systems Phys. Rev. E $\mathbf{5 1} 980$

[3] Kurths J, Boccaletti S, Grebogi C and Lai Y C 2003 Introduction: control and synchronization in chaotic dynamical systems Chaos 13126

[4] Schafer C, Rosenblum M G, Abel H H and Kurths J 1999 Synchronization in the human cardiorespiratory system Phys. Rev. E 60857

[5] Ott E 2002 Chaos in Dynamical Systems (Cambridge: Cambridge University Press)

[6] Munk M H J, Roelfsema P R, König P, Engel A K and Singer W 1996 Role of reticular activation in the modulation of intracortical synchronization Science 272 271-4

[7] Singer W 1999 Neuronal synchrony: a versatile code for the definition of relations? Neuron 24 49-65

[8] Sauvé K 1999 Gamma-band synchronous oscillations: recent evidence regarding their functional significance Consc. Cogn. $8213-24$

[9] Gray C M 1999 The temporal correlation hypothesis of visual feature integration: still alive and well Neuron 24 31-47

[10] von der Malsburg C 1985 Nervous structures with dynamical links Ber. Bunsenges. Phys. Chem. 89 $703-10$

[11] Bi G Q and Poo M M 1998 Synaptic modifications in cultured hippocampal neurons: dependence on spike timing, synaptic strength, and postsynaptic cell type Nature $\mathbf{4 0 1} 792$ 
[12] McMahon D B T and Barrionuevo G 2002 Short- and long-term plasticity of the perforant path synapse in hippocampal area ca3 J. Neurophysiol. 88528

[13] Song S and Abbott L F 2001 Cortical development and remapping through spike timing-dependent plasticity Neuron 32339

[14] Rosenblum M G, Pikovsky A S and Kurths J 1997 From phase to lag synchronization in coupled chaotic oscillators Phys. Rev. Lett. 784193

[15] Rosenblum M G, Pikovsky A S and Kurths J 1996 Phase synchronization in chaotic oscillators Phys. Rev. Lett. 761804

[16] Rosenblum M G, Pikovsky A S, Osipov G and Kurths J 1997 Phase synchronization of chaotic oscillators by external driving Physica D 104219

[17] Parlitz U, Junge L, Lauterborn W and Kocarev L 1996 Experimental observation of phase synchronization Phys. Rev. E $542115-7$

[18] Zhou C, Kurths J, Kiss I Z and Hudson J L 2002 Noise-enhanced phase synchronization of chaotic oscillators Phys. Rev. Lett. 89014101

[19] Pazo D, Zaks A M and Kurths J 2003 Role of unstable periodic orbits in phase and lag synchronization between coupled chaotic oscillators Chaos 13309

[20] Shannon C E 1949 The Mathematical Theory of Communication (Chicago: University of Illinois Press)

[21] Schurmann T and Grassberger P 1996 Entropy estimation of symbol sequences Chaos 6414

[22] Schouten J C, Takens F and van den Bleek C M 1994 Maximum-likelihood estimation of the entropy of the attractor Phys. Rev. E 49126

[23] Macek W M and Redaelli S 2000 Estimation of the entropy of the solar wind flow Phys. Rev. E 626496

[24] Bezerianos A, Tong S and Thakor N 2003 Time-dependent entropy estimation of EEG rhythm changes following brain ischemia Ann. Biomed. Eng. 31221

[25] Pecora L M and Carroll T L 1990 Synchronization in chaotic systems Phys. Rev. Lett. 64821

[26] Kocarev L and Parlitz U 1996 Synchronizing spatiotemporal chaos in coupled nonlinear oscillators Phys. Rev. Lett. 772206

[27] Gerstner W 1996 Rapid phase locking in systems of pulse-coupled oscillators with delays Phys. Rev. Lett. 76 $1755-58$

[28] Luthi A and McCormick D A 1998 H-current: properties of a neuronal and network peacemaker Neuron 21 9-12

[29] Rössler O E 1976 An equation of continuous chaos Phys. Lett. A 57397

[30] Hindmarsh J L and Rose R M 1994 A model for rebound bursting in mammalian neurons Phil. Trans. R. Soc. B 346129

[31] Hindmarsh J L and Rose R M 1994 A model of intrinsic and driven spindling in thalamocortical neurons Phil. Trans. R. Soc. B 346165

[32] Hindmarsh J L and Rose R M 1994 Resonance in a model of a mammalian neuron Phil. Trans. R. Soc. B 346 151 